\title{
Catalpol Ameliorates Podocyte Injury by Stabilizing Cytoskeleton and Enhancing Autophagy in Diabetic Nephropathy
}

\author{
Yan Chen ${ }^{1}$, Qingpu Liu', Zengfu Shan ${ }^{1}$, Wangyang Mi', Yingying Zhao', Meng Li', \\ Baiyan Wang ${ }^{2}$, Xiaoke Zheng ${ }^{1,3 *}$ and Weisheng Feng ${ }^{1,3 *}$ \\ ${ }^{1}$ College of Pharmacy, Henan University of Chinese Medicine, Zhengzhou, China, ${ }^{2}$ College of Basic Medicine, Henan University \\ of Chinese Medicine, Zhengzhou, China, ${ }^{3}$ Co-Construction Collaborative Innovation Center for Chinese Medicine and \\ Respiratory Diseases by Henan \& Education Ministry of P.R. China, Henan University of Chinese Medicine, Zhengzhou, China
}

OPEN ACCESS

Edited by:

Jiahong Lu,

University of Macau, China

Reviewed by:

Yanggang Yuan,

Naniing Medical University,

China

Min Li,

Sun Yat-sen University, China

${ }^{*}$ Correspondence:

Xiaoke Zheng

zhengxk.2006@163.com

Weisheng Feng

fwsh@hactcm.edu.cn

Specialty section:

This article was submitted to

Ethnopharmacology,

a section of the journal

Frontiers in Pharmacology

Received: 28 August 2019 Accepted: 13 November 2019 Published: 10 December 2019

Citation:

Chen Y, Liu Q, Shan Z, Mi W, Zhao Y, Li M, Wang B, Zheng $X$ and Feng W (2019) Catalpol Ameliorates

Podocyte Injury by Stabilizing

Cytoskeleton and Enhancing Autophagy in Diabetic Nephropathy.

Front. Pharmacol. 10:1477.

doi: 10.3389/fphar.2019.01477
Catalpol, an iridoid glycoside extracted from Rehmannia glutinosa, has been found to ameliorate diabetic nephropathy ( $\mathrm{DN})$, but the mechanism has not been clarified. Podocyte injury play a key role in the pathogenesis of DN. This study mainly investigated the protective effect and potential mechanism of catalpol on podocyte injury of DN in vivo and in vitro. The results indicated that the pathological features of DN in mice were markedly ameliorated after treatment with catalpol. Moreover, podocyte foot process effacement, and down-regulation of nephrin and synaptopodin expression in DN mice were also significantly improved after treatment with catalpol. In vitro, catalpol rescued disrupted cytoskeleton and increased migration ratio in podocytes induced by high glucose, the effect might be attributable to the inhibition of RhoA and Cdc42 activities but not Rac1. Furthermore, the impaired podocyte autophagy in DN mice was significantly enhanced after catalpol treatment. And catalpol also enhanced autophagy and lysosome biogenesis in cultured podocytes under high glucose condition. In addition, we found that catalpol could inhibit mTOR activity and promote TFEB nuclear translocation in vivo and in vitro experiments. Our study demonstrated that catalpol could ameliorate podocyte injury in DN, and the protective effect of catalpol might be attributed to the stabilization of podocyte cytoskeleton and the improvement of impaired podocyte autophagy.

Keywords: catalpol, diabetic nephropathy, podocyte injury, autophagy, cytoskeleton

\section{INTRODUCTION}

Diabetic nephropathy (DN) is a common diabetic microvascular complication, which has been one of the leading causes of end-stage renal disease and increases the risks of cardiovascular disease events and death (Georgianos and Agarwal, 2017; Cho et al., 2018; Ravindran et al., 2019). Intensive glycemia control and treatments for hypertension are established therapies toward DN (Fineberg et al., 2013), despite proven effective therapies, they are not enough to arrest the development of DN to end-stage renal disease and there is, thus, the great need to develop additional therapies.

Albuminuria is the common clinically manifestation of DN that usually develops before the glomerular filtration rate (GFR) impaired and increases the risk of decline of GFR (Molitch et al., 2010), the prevention of albuminuria is therefore a recommendatory therapy in patients with DN 
(Looker et al., 2019). Podocyte play a key role in the pathogenesis of DN (Denhez et al., 2019). Multiple clinical studies have confirmed that podocyte foot process effacement is found in DN patients, and such podocyte injury is accompanied with abnormal glomerular filtration function (Shankland, 2006; George et al., 2012; Hara et al., 2019). As highly differentiated cells, podocytes line the urinary side of the glomerular basement membrane, which are essential for maintenance of the structure and function of the glomerular filtration barrier through their interdigitating foot processes (Nagata, 2016). This unique function of podocytes depends on their complex cytoskeleton structure, particularly the actin-rich foot processes. Dynamic regulation of the podocyte foot process cytoskeleton plays a critical role in maintaining sustained glomerular filter function, and podocyte injury is accompanied by the cytoskeleton rearrangement, which is closely associated with foot process effacement and subsequent proteinuria (Tryggvason et al., 2006; Wiggins, 2007; Jefferson et al., 2011; Toffoli et al., 2018). Given the key role of cytoskeleton dynamics, several previous studies have revealed that improving cytoskeleton dynamics would ameliorate DN (Kikuchi et al., 2007; Reiser and Sever, 2013; Lin and Susztak, 2016).

Autophagy is a highly conserved intracellular catabolic process for degradation of proteins and organelles via the lysosomal pathway (Mizushima and Komatsu, 2011; Mizushima et al., 2008). As terminally differentiated cells, podocytes exhibit a high level of basal autophagy, which play a pivotal role for maintaining podocyte's homeostasis (Hartleben et al., 2010; Yasuda-Yamahara et al., 2015; Jin et al., 2019). Previous studies have shown that podocyte autophagy insufficiency is found in diabetic patients accompanied by massive proteinuria, and impaired podocyte autophagy exacerbated proteinuria in $\mathrm{DN}$, these studies indicate the importance of podocyte autophagy in the pathogenesis of DN (Kume and Koya, 2015; Lenoir et al., 2015; Tagawa et al., 2016). Recently, transcription factor EB (TFEB) was identified to regulated the transcription of various genes involved in autophagy and lysosomal biogenesis, and inhibition of the mammalian target of rapamycin (mTOR) has been shown to protect podocyte injury by promoting nuclear translocation of TFEB in animal models of DN (Settembre et al.,2011; Settembre et al., 2013).

Catalpol (Figure 1A) is a natural iridoid glycoside compound derived from traditional Chinese medicinal herb Rehmannia glutinosa. It has been demonstrated that catalpol possesses a wide range of biological activities, including anti-diabetic, antiinflammatory and neuroprotective effect (Chen et al., 2013; Liu et al., 2018; Yan et al., 2018). Additionally, previous study
A

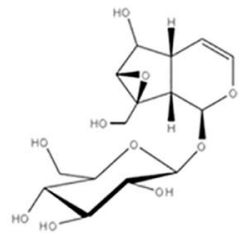

C

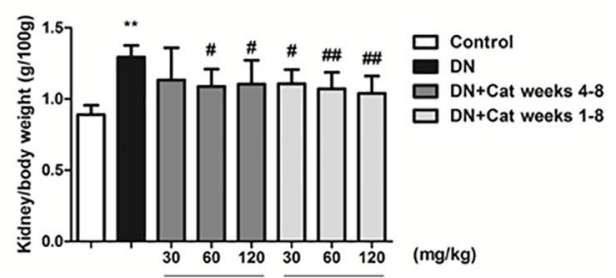

$\mathbf{E}$

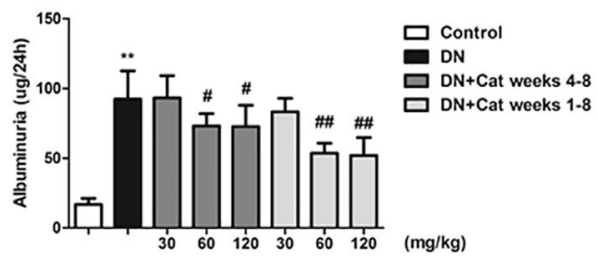

B

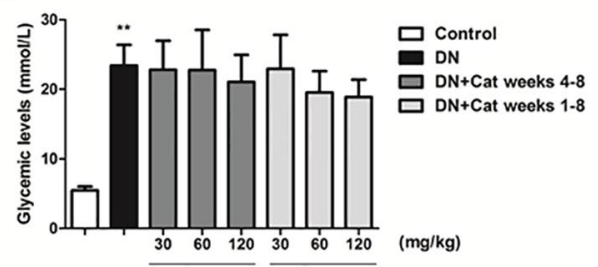

D

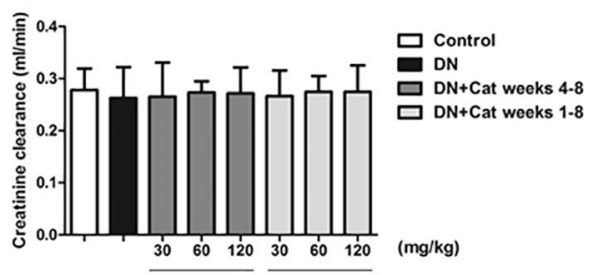

F

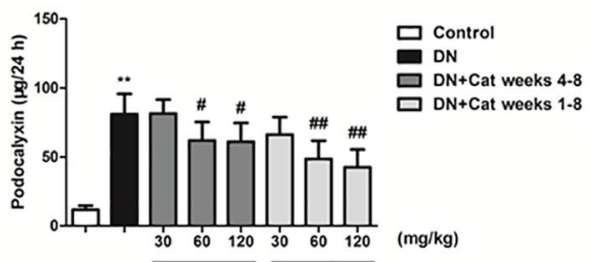

FIGURE 1 | Effects of catalpol on physiological and renal functional parameters in diabetic nephropathy (DN) mice. (A) Chemical structure of catalpol. (B) The level of blood glucose in Control, DN, and DN plus catalpol-treated (30,60, and 120 mg/kg, weeks 4-8 and weeks 1-8) mice $(\mathrm{n}=8)$. (C) Kidney/body weight in Control, $\mathrm{DN}$, and DN plus different doses of catalpol-treated mice $(n=8)$. (D) Creatinine clearance in Control, DN, and DN plus different doses of catalpol-treated mice $(n=$ 8). (E) Urinary albumin excretion in Control, DN, and DN plus different doses of catalpol-treated mice $(n=8)$. (F) Urinary podocalyxin level in Control, $D N$, and $D N$ plus different doses of catalpol-treated mice $(n=8)$. Data represent the mean values \pm SD. ${ }^{* *} p<0.01$ vs Control, ${ }^{*} p<0.05,{ }^{* \#} p<0.01$ vs $D N$. 
reported that catalpol could ameliorate renal function and proteinuria, which was closely associated with podocyte injury in DN (Dong and Chen, 2013). However, it is unclear whether catalpol exhibits a beneficial effect on podocyte injury in DN. In the present study, we investigated the effect and mechanism of catalpol on podocyte injury in $\mathrm{DN}$, and found that catalpol could ameliorate podocyte injury through stabilizing cytoskeleton and enhancing autophagy in DN.

\section{MATERIALS AND METHODS}

\section{Materials}

Catalpol (98\%) was purchased from Nanjing Spring \& Autumn Biological Engineering Co., Ltd. (Jiangsu, China). Primary antibodies directed against the following proteins were used: Anti-synaptopodin (sc-515842) was purchased from Santa Cruz Biotechnology (CA, United States). Anti-nephrin (ab216341), anti-RhoA (ab187027), anti-Cdc42 (ab187643), anti-Rac1 (ab180683), and anti-LC3B (ab48394) antibodies were purchased from Abcam (Cambridge, MA, USA). Anti-p62 (\#23214), antip70s6k (\#9202), anti-phospho-p70s6k (\#9234), and anti-histone H3 (\#4499) antibodies were purchased from Cell Signaling Technology (Danvers, MA, USA). Anti-TFEB (A303-673A) was purchased from Bethyl Laboratories, Inc (Montgomery, MA, USA). Anti- $\beta$-actin (AC026) was purchased from Abclonal (Boston, MA, USA).

\section{Animals Experimental Design}

Male C57BL/6J mice (8 weeks) were obtained from the Beijing Vital River Laboratory Animal Technology Co., Ltd. (Beijing, China). Mice were housed in the central animal facility of the Henan University of Chinese Medicine and maintained on a normal diet under standard animal housing condition (temperature $25 \pm 1{ }^{\circ} \mathrm{C}$ and humidity $50 \% \pm 10 \%$ with a $12-\mathrm{h}$ dark/light cycle). After 7 days of acclimation, mice were intraperitoneal injection with streptozotocin in citrate buffer $\mathrm{pH} 4.5$ at a dose $170 \mathrm{mg} / \mathrm{kg}$ of body weight to establish the diabetic model. Catalpol (Cat) $(30,60,120 \mathrm{mg} / \mathrm{kg})$ or vehicle was given by gavage once a day from weeks $4-8$ or weeks $1-8$ after streptozotocin administration $(\mathrm{n}=8)$. Control nondiabetic mice were administered vehicle daily. All treatments continued for 8 weeks. At the end of experiment, the mice were anesthetized with $1.5 \%(\mathrm{w} / \mathrm{v})$ pentobarbital sodium solution, then kidney tissues and blood samples were collected for further experiments. All animal experiments were approved by the Institutional Animal Care and Research Ethics Committee of Henan University of Chinese Medicine and confirmed to the guidelines of the National Institute of Health for the Care and Use of Laboratory Animals.

\section{Cell Culture}

Conditionally immortalized mouse podocytes were provided by National Infrastructure of Cell Line Resource (Beijing, China) and described in detail previously (Mundel et al., 1997). Podocytes were cultured in RPMI 1640 medium (Life Technologies, Grand
Island, NY) supplemented with $10 \%$ fetal bovine serum, $100 \mu \mathrm{g} /$ $\mathrm{ml}$ streptomycin, and $100 \mathrm{U} / \mathrm{ml}$ penicillin. Recombinant mouse interferon- $\gamma$ (50 U/ml, PeproTech, California, USA) was added to culture medium at $33^{\circ} \mathrm{C}$ in a humidified atmosphere of $5 \%$ $\mathrm{CO}_{2}$. To induce differentiation, podocytes were cultured in RPMI 1640 medium without IFN- $\gamma$ at $37^{\circ} \mathrm{C}$ for 14 days. When podocytes were well-differentiated, they were incubated with normal glucose (NG) media $(5.5 \mathrm{mmol} / \mathrm{L}$ glucose $+34.5 \mathrm{mmol} / \mathrm{L}$ mannitol) or high glucose (HG) media ( $40 \mathrm{mmol} / \mathrm{L}$ glucose) with or without catalpol $(1,5,10 \mu \mathrm{mol} / \mathrm{L})$ for $48 \mathrm{~h}$ and collected for the following assays.

\section{Physiological Parameters}

Fasting blood glucose levels were measured by using a Glucometer (OMRON Corporation, Tokyo, Japanese). For urine collection, mice were held in a metabolic cage for $24 \mathrm{~h}$. Levels of urinary albuminuria and podocalyxin were measured using ELISA kits (Elabscience, Wuhan, China). Creatinine was tested by a commercial assay kit (Jiancheng, Jiangsu, China).

\section{Histology and Immunohistochemistry}

Kidney tissues from mice were fixed in $4 \%$ buffered paraformaldehyde for 2 days, embedded in paraffin, and processed for sectioning. Kidney sections were stained via periodic acidSchiff staining (PAS), mesangial area was analyzed from digital pictures of 20-25 glomeruli per group using Image-Pro plus software. For immunohistochemistry, nephrin expression was detected on $3-\mu \mathrm{m}$ paraffin-embedded kidney sections. After incubated in citrate antigen retrieval solution, sections were incubated with $\mathrm{H}_{2} \mathrm{O}_{2}$ to inhibited the endogenous peroxidase activity. After blocking with BSA, the sections were incubated with anti-nephrin antibody overnight at $4^{\circ} \mathrm{C}$, then exposed to secondary antibody, horseradish peroxidase-labeled swine antirabbit IgG (Servicebio, Wuhan, China) for $1 \mathrm{~h}$, specific labeling of nephrin was performed with the DAB substrate kit (Servicebio, Wuhan, China). Immunohistochemical quantification was expressed as the percentage of positive staining area using ImagePro plus software.

\section{Transmission Electron Microscopy}

Small fragments of renal cortex were fixed in glutaraldehyde and osmic acid, dehydrated with graded ethanol, and embedded in ethoxyline resin. Sections 70 to $80 \mathrm{~nm}$ thick were cut and viewed under transmission electron microscopy (TEM) (JEM-1400, JEOL, Japanese).

\section{Immunofluorescence}

For immunofluorescence, fixed podocytes or paraffin-embedded kidney sections were labeled with primary antibody against synaptopodin or TFEB and then incubated with an FITCconjugated anti-mouse antibody or AlexaFluor555-conjugated anti-rabbit antibody (Beyotime, Shanghai, China). F-actin was labeled with rhodamine phalloidin (Cytoskeleton Inc., Denver, USA). The slides were viewed using a confocal microscope (Olympus Corporation, Tokyo, Japan). 


\section{Podocyte Migration Assays}

After different treatments, cultured podocytes were scratched with a pipette. Images of the wounded area were taken on an inverted microscope at indicated time points and were analyzed using Image $\mathrm{J}(\mathrm{NIH}$, Bethesda, MD). The percentage of cell migration area was calculated as Area $0 \mathrm{~h}$ - Area $24 \mathrm{~h}$ /Area $0 \mathrm{~h}$. RTCA xCELLigence system (Roche Applied Science) was used to realtime migration assay according to the manufacturer's instruction. Briefly, at the beginning, the lower chamber was filled with $10 \%$ fetal bovine serum and different treatments (NG, HG, or HG with different concentrations of catalpol), and $5 \times 10^{4}$ cells were plated in the upper chamber with serum-free media and different treatments. The data were exported from RTCA software.

\section{Pull-Down Assay for Small GTPases Activities}

RhoA, Cdc42, and Rac1 activities were determined by measuring Rhoketin RBD beads or PAK-PBD beads pulled down by GTP-RhoA and GTP-Cdc42/Rac1, respectively (Garcia-Mata et al., 2006). RhoA, Cdc42, and Rac1 were separated on 12\% SDS-PAGE following the manufacturer's instructions (Cytoskeleton Inc., Denver, USA).

\section{Western Blot}

The proteins from prepared renal cortex or cultured podocytes were quantified with BCA protein Assay kit (Solarbio Life Sciences, Beijing, China). Proteins were separated by $8 \%-15 \%$ SDS-PAGE and transferred onto polyvinylidene difluoride membrane. After blocking with 5\% non-fat milk for $1 \mathrm{~h}$, the membranes were incubated with primary antibodies against synaptopodin (1:500 dilution), RhoA (1:2000 dilution), Cdc42 (1:5000 dilution), Rac1 (1:1000 dilution), LC3B (1:1000 dilution), p62 (1:1000 dilution), p-p70s6k (1:1000 dilution), p70s6k (1:1000 dilution), TFEB (1:1000 dilution), histone $\mathrm{H3}$ (1:1000 dilution), and $\beta$-actin (1:5000 dilution) overnight at $4^{\circ} \mathrm{C}$. After being washed five times in TBST, the membranes were treated for $1 \mathrm{~h}$ with IRDyeIgG (1:5000 dilution) and washed five times in TBST again. Finally, the protein bands were visualized by Odyssey Infrared Imager (LI-COR Biosciences, USA) and quantified by using Image Studio software. The protein levels were normalized against the $\beta$-actin or histone $\mathrm{H} 3$.

\section{Transfection of Adenovirus}

mRFP-GFP-LC3B adenovirus was used to detect the autophagy in cultured podocytes (Ginet et al., 2014). In brief, after incubating with mRFP-GFP-LC3B adenovirus for $6 \mathrm{~h}$ in RPMI 1640 medium, the medium was removed and replaced to NG medium ( $5.5 \mathrm{mmol} / \mathrm{L}$ glucose $+34.5 \mathrm{mmol} / \mathrm{L}$ mannitol) or HG medium (40 mmol/L glucose) with or without catalpol (1, 5, 10, $\mu \mathrm{mol} / \mathrm{L}$ ). Confocal images were acquired after $48 \mathrm{~h}$ treatment as described in podocyte culture using FV1200 biological confocal laser scanning microscope (Olympus Corporation, Japanese).

\section{Data Analysis}

Statistical analyses were performed by using GraphPad Prism 5.0 software (GraphPad Software ${ }^{\circledR}$, San Diego, CA, USA). The results were presented as mean \pm SD. Statistical significance was assessed by one-way ANOVA followed by Dunnett's post hoc test. Differences with $p<0.05$ was considered as significant.

\section{RESULTS}

\section{Effects of Catalpol on Physiological and Renal Functional Parameters in DN Mice}

As shown in Figure 1B, the level of blood glucose was significantly higher in DN compared to control mice, treatment with catalpol did not reverse the change. The kidney/body weight ratio in DN mice was significantly increased compared to that in control mice, treatment with catalpol at doses of 60 and $120 \mathrm{mg} / \mathrm{kg}$ (weeks 4-8) or at doses of 30,60 , and $120 \mathrm{mg} / \mathrm{kg}$ (weeks 1-8) significantly decreased the kidney/body weight ratio (Figure 1C). There was only a non-significant decline in creatinine clearance in DN mice compared to control mice (Figure 1D). At the end of the treatment period, urinary albumin excretion was significantly increased in DN mice compared to control mice, treatment with catalpol at doses of 60 and $120 \mathrm{mg} / \mathrm{kg}$ (weeks 4-8 and weeks 1-8) significantly reduced urinary albumin excretion (Figure 1E). In accordance with the results on albuminuria, DN mice exhibited a significant increase in the level of podocalyxin in urine compared to control mice, treatment with catalpol at doses of 60 and 120 $\mathrm{mg} / \mathrm{kg}$ (weeks 4-8 and weeks 1-8) attenuated the increase in the level of podocalyxin in urine (Figure 1F).

\section{Effect of Catalpol on Renal Histological Changes in DN Mice}

Histological changes in DN patients were characterized by glomerular hypertrophy and mesangial expansion (Li et al., 2007). Kidney PAS staining showed that DN mice revealed remarkable mesangial expansion, treatment with catalpol at doses of 60 and $120 \mathrm{mg} / \mathrm{kg}$ (weeks $4-8$ and weeks $1-8$ ) had a significant decrease in PAS-positive matrix (Figure 2).

\section{Effect of Catalpol on Podocyte Injury in DN Mice}

Podocyte foot process effacement is considered as one of the early clinical manifestations, which strongly correlated with albuminuria and decreased GFR in DN patients (Lin and Susztak, 2016). As shown in Figures 3A, B, abnormal morphology and segmental effacement of podocyte foot processes were observed in DN mice by TEM, while treatment with catalpol at doses of 30,60 and $120 \mathrm{mg} / \mathrm{kg}$ (weeks 1-8) could ameliorate foot processes effacement. Next, we measured podocyte injury by immunohistochemistry and immunofluorescence with nephrin and synaptopodin, two podocyte-specific markers. As shown in Figures 3A, C, D, there was a significant reduction in both nephrin-positive and synaptopodin-positive area in glomeruli of $\mathrm{DN}$ mice relative to control mice, the reduction was significantly ameliorated in catalpol-treated $(60,120 \mathrm{mg} / \mathrm{kg}) \mathrm{DN}$ mice. In keeping with the results on immunofluorescence, the data were confirmed by measuring synaptopodin expression in renal cortex by western blotting (Figures 3E, F). 


\section{A}
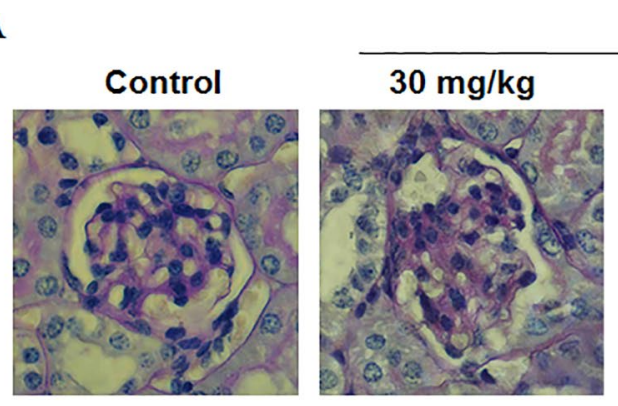

\section{DN+Cat weeks $4-8$}
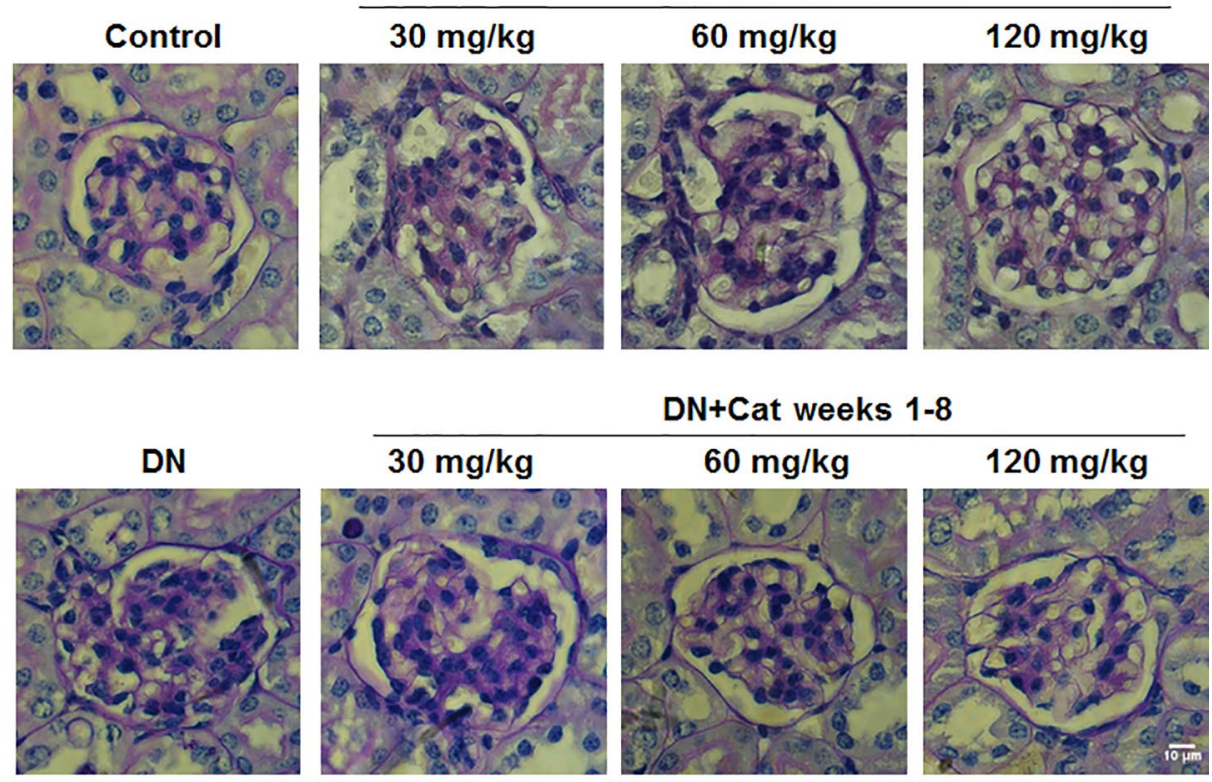

DN+Cat weeks 1-8

B

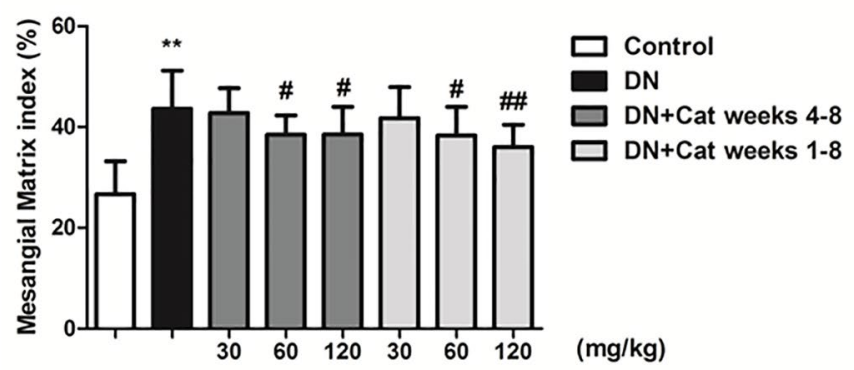

FIGURE 2 | Effect of catalpol on renal histological changes in DN mice. (A) Representative images of periodic acid-Schiff (PAS) staining in glomeruli from Control, DN, and DN plus catalpol-treated (30, 60, and 120 mg/kg, weeks 4-8 and weeks 1-8) mice ( $\mathrm{n}=8)$. Scale bar: 10 um. (B) Percentage of PAS glomerular-positive areas from Control, DN, and DN plus different doses of catalpol ( $n=8,20-25$ images from each group). Data represent the mean values $\pm \mathrm{SD}$. ${ }^{\star *} p<0.01$ vs Control, " $p<0.05$, \# $p<0.01$ vs DN.

\section{Effects of Catalpol on Cytoskeleton and Migration in Podocytes Exposed to HG}

Podocytes were treated with different concentrations $(1,5,10 \mu \mathrm{M})$ of catalpol in NG or HG medium in the MTT test. As shown in Figure $\mathbf{4 A}$, treatment with catalpol did not alter cell viability compared to that of podocytes treated with NG. HG treatment significantly decreased cell viability in podocytes compared to that in cells treated with NG, however, treatment with catalpol significantly increased cell viability in podocytes incubated with HG (Figure 4B).

Cytoskeletal structure is critical to podocyte foot processes and is essential for maintaining the integrity of the glomerular filtration barrier (Lal and Patrakka, 2018), we next observed cytoskeletons using rhodamine phalloidin staining by confocal microscope. As expected, exposure of cultured podocytes to HG significantly disrupted podocyte F-actin stress fiber cytoskeletal structure, treatment with catalpol at concentrations of 5 and $10 \mu \mathrm{M}$ could rescue the disruption of F-actin stress fiber in podocytes exposed to HG (Figure 4C).
Then, we detected the effect of catalpol on podocyte migration using wound healing assay and RTCA. As shown in Figures 4D, E, wound healing assay showed that podocytes with high concentration of glucose showed a significantly accelerated wound healing kinetics compared to NG group, the migration rates of podocytes were remarkably decreased by treatment with catalpol at concentrations of 1,5 and $10 \mu \mathrm{M}$. The RTCA experiment showed that catalpol treatment reversed the increased migration ratio induced by $\mathrm{HG}$, which was in accordance with the wound healing assay (Figure 4F).

\section{Effect of Catalpol on the Activities of RhoA, Cdc42, and Rac1 in Podocytes Exposed to HG}

Given that the pivotal role of Rho family of GTPases in cytoskeletal dynamics, we further detected the activities of RhoA, Cdc42, and Rac1 in culture podocytes by GST pull-down 


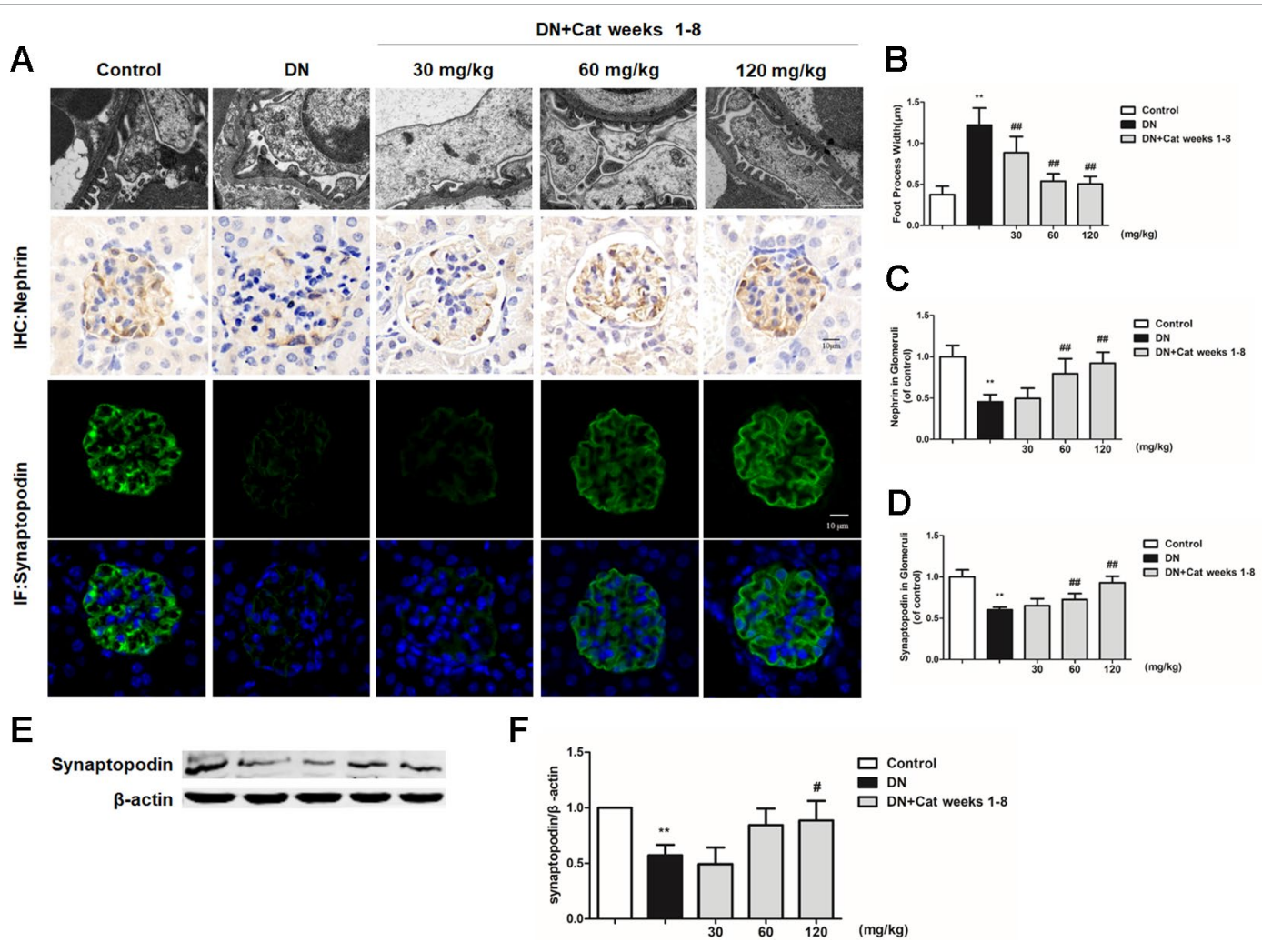

FIGURE 3 | Effect of catalpol on podocyte injury in DN mice. (A) Representative transmission electron microscopy (TEM) images of podocyte foot processes in Control, DN, and DN plus catalpol-treated (30,60, and $120 \mathrm{mg} / \mathrm{kg}$, weeks 1-8) mice $(\mathrm{n}=3)$. Scale bar: $1 \mu \mathrm{m}$. Representative images of glomeruli showing nephrinpositive and synaptopodin-positive expression in glomeruli of Control, DN, and DN plus different doses of catalpol-treated mice $(\mathrm{n}=8)$. Scale bar: $10 \mu \mathrm{m}$.

(B) Quantification of podocyte foot process width by electron micrographs ( $n=3,10$ images from each group). (C) Quantification of results for immunohistochemical staining of nephrin expressed as the percentage of stained area in glomeruli ( $n=8,20-25$ images from each group). (D) Quantification of results in synaptopodin in glomeruli by the ratio of integrated optical density (IOD) to area $(n=8,21-25$ images from each group). (E) Western blotting for expression of synaptopodin in Control, DN, and DN plus different doses of catalpol-treated mice $(n=3)$. (F) Quantification of results in panel E. Data represent the mean values \pm SD. ${ }^{* *} p<0.01$ vs Control, $\# p<0.05, \# p<0.01$ vs DN.

assay (Huang et al., 2018; Pan et al., 2018). Our results revealed no significant difference in the Racl activation levels between NG and HG group but a notable augmentation of the RhoA-GTP level and Cdc42-GTP level in the HG group compared with those in the NG group, treatment with catalpol at doses of 5 and $10 \mu \mathrm{M}$ inhibited the RhoA and Cdc42 activities, suggesting that catalpol maintained stable podocyte cytoskeleton through controlling RhoA and Cdc42 activities (Figure 5).

\section{Effect of Catalpol on Podocyte Autophagy in DN Mice}

To explore podocyte autophagy in DN mice, we examined the number of autophagic vacuoles in podocytes by TEM. We observed that the number of autophagic vacuoles was significantly decrease in DN mice compared to control mice, treatment with catalpol at doses of 60 and $120 \mathrm{mg} / \mathrm{kg}$ (weeks 1-8) significantly increased the number of autophagic vacuoles (Figures 6A, B). p62, a selective substrate of autophagy, which was found abnormal accumulation in the glomeruli of DN patients (Tagawa et al., 2016; Lin et al., 2019). There was a significant decrease in the autophagy marker LC3B expression and increase in p62 expression of DN mice which was ameliorated by catalpol treatment at doses of 60 and $120 \mathrm{mg} / \mathrm{kg}$ (Figures 6C, D).

\section{Effect of Catalpol on Autophagy in Podocytes Exposed to HG}

In Ad-mRFP-GFP-LC3B-transfected podocytes, high concentration of glucose induced the decrease of mRFP fluorescence, indicating autophagy insufficiency, in contrast, treatment with catalpol $(10 \mu \mathrm{M})$ increased the number of mRFP fluorescence puncta, indicating the enhancing autophagy (Figure 7A). Furthermore, the decreased LC3B expression and increased p62 expression induced by HG were remarkably reversed by treatment with catalpol at concentrations of 5 and $10 \mu \mathrm{M}$ (Figures 7B, C).

\section{Effects of Catalpol on mTOR/TFEB Pathway in DN Mice and Podocytes Exposed to HG}

To further explore the possible mechanism underlying the enhanced effect of catalpol on impaired podocyte autophagy, we determined mTOR activity and TFEB nuclear translocation 
A

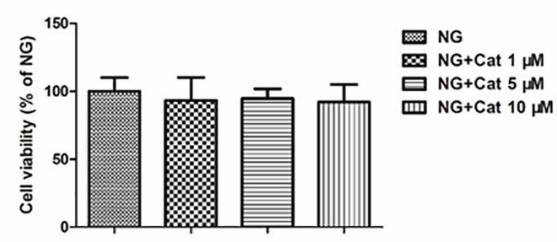

B

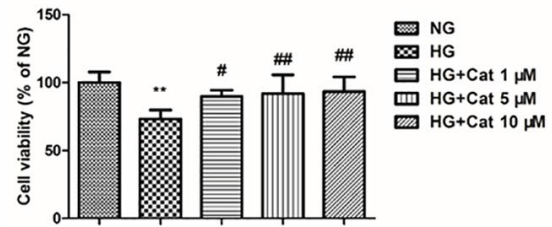

C

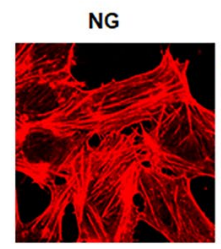

HG

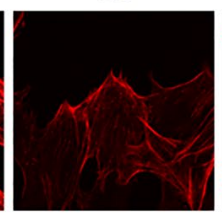

$\mathrm{HG}+\mathrm{C}$ at $1 \mu \mathrm{M}$
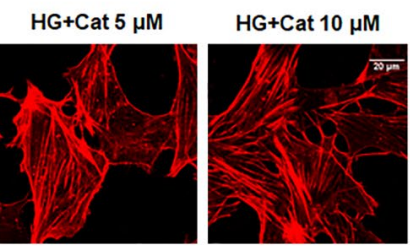

D
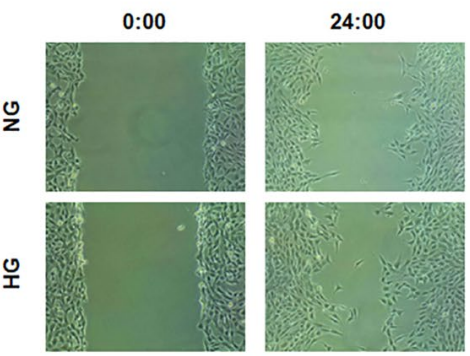

E
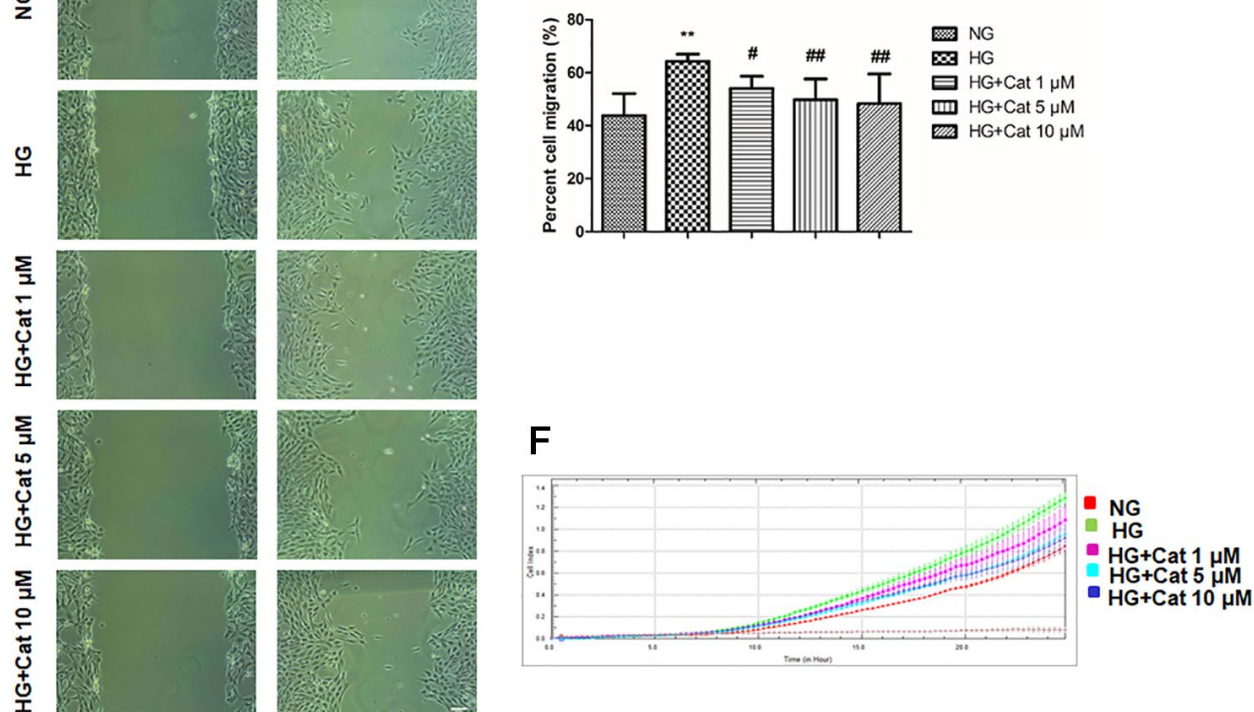

FIGURE 4 | Effects of catalpol on cytoskeleton and migration in podocytes exposed to high glucose. (A) Cell viability was determined by MTT. Podocytes were cultured with normal glucose (NG) $(5.5 \mathrm{mmol} / \mathrm{L})$ and $\mathrm{NG}$ plus different concentrations of catalpol $(1,5,10 \mu \mathrm{M})$ for $48 \mathrm{~h}$. (B) Cell viability was determined by MTT. Podocytes were cultured with $N G(5.5 \mathrm{mmol} / \mathrm{L})$, high glucose $(\mathrm{HG})(40 \mathrm{mmol} / \mathrm{L})$, and $\mathrm{HG}$ plus different concentrations of catalpol $(1,5,10 \mu \mathrm{M})$ for $48 \mathrm{~h}$.

(C) Rhodamine phalloidin staining of podocytes. Scale bar: $20 \mu \mathrm{m}$. (D) The images were recorded immediately $(0 \mathrm{~h})$ and at $24 \mathrm{~h}$ after scratch $(\mathrm{n}=3)$. Scale bar: 200 $\mu \mathrm{m}$. (E) Quantification of the cell migration area. ( $n=3,10$ areas from each group). (F) Migration assay using the RTCA xCELLigence system (detailed in Materials and Methods). Data represent the mean values \pm SD, ${ }^{* *} p<0.01$ vs NG, ${ }^{*} p<0.05,{ }^{* \#} p<0.01$ vs HG.

in DN mice and cultured podocytes. As shown in Figure 8A, western blotting revealed that phosphorylated p70s6k (p-p70s6k) were significantly increased in DN mice compared with that in control mice, however, treatment with catalpol at doses of 30 , 60 , and $120 \mathrm{mg} / \mathrm{kg}$ (weeks 1-8) significantly decreased p-p70s6k expression. In vitro, exposure of cultured podocytes to $\mathrm{HG}$ induced a significant increase in p-p70s6k expression, treatment with catalpol at concentrations of 5 and $10 \mu \mathrm{M}$ decreased the expression of p-p70s6k (Figure 8B). Since inhibition of mTOR activity could promote TFEB nuclear translocation (Settembre et al., 2012; Zhao et al., 2018; Ye et al., 2019), we next determined whether catalpol could activate TFEB and promote its nuclear translocation. As shown in Figure 8C, catalpol treatment at concentrations of 5 and $10 \mu \mathrm{M}$ showed an obviously effect on TFEB nuclear translocation in cultured podocytes incubated with HG. In vivo, western blotting showed that treatment with catalpol $(60,120 \mathrm{mg} / \mathrm{kg})$ significantly rescued the downregulation of nucleus TFEB expression in DN mice, suggesting that catalpol promoted TFEB nuclear translocation in DN mice (Figures 8D, E).

\section{DISCUSSION}

Podocyte injury play a key role in the progression of DN (dai et al., 2017; Kim et al., 2018). In the present study, we provided 
A

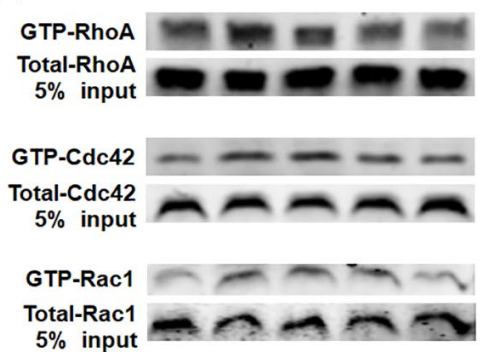

B

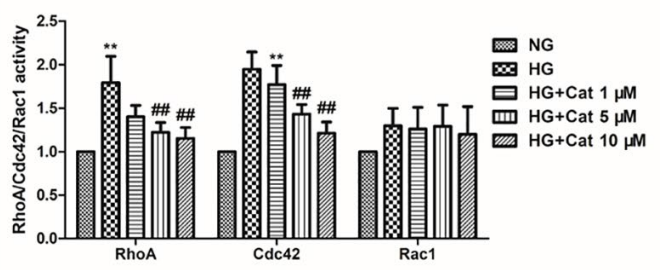

FIGURE 5 | Effects of catalpol on the activities of RhoA, Cdc42, and Rac1 in podocytes exposed to high glucose. (A) Active GTP-bound forms of RhoA, Cdc42, and Rac1 purified from podocytes cultured with NG (5.5 mmol/L), HG (40 mmol/L), and HG plus different concentrations of catalpol (1, 5, $10 \mu \mathrm{M})$ by GST pull-down assays and subjected to western blotting $(n=3)$. (B) Quantification of results in panel A. Data represent the mean values \pm SD from three independent experiments, ${ }^{* *} p<0.01$ vs NG, ${ }^{\#} p<0.01$ vs HG.

\section{A}
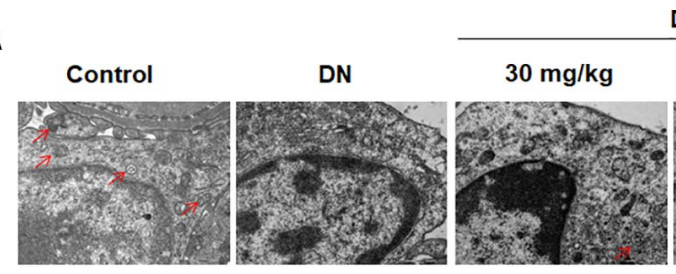

DN+Cat weeks $1-8$

B

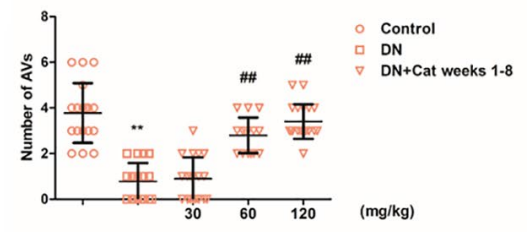

\section{C}
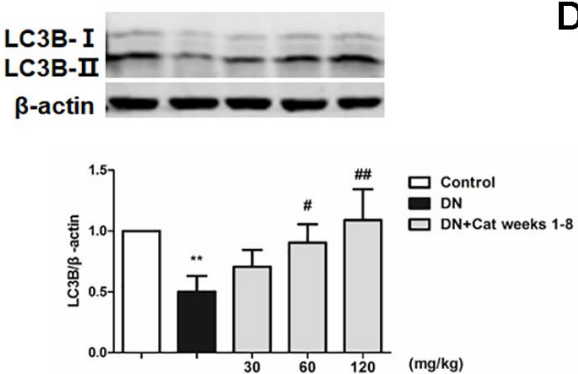

D
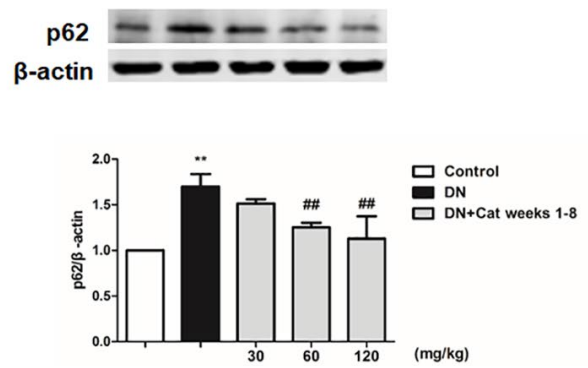

FIGURE 6 | Effect of catalpol on podocyte autophagy in DN mice. (A) Representative TEM images of autophagic vacuoles (red arrow) in Control, DN, and DN plus catalpol-treated $(30,60$, and $120 \mathrm{mg} / \mathrm{kg}$, weeks $1-8)$ mice $(n=3)$. Scale bar: $1 \mu \mathrm{m}$. (B) The numbers of autophagic vacuoles by electron micrographs $(n=3,15-20$ electron micrographs were selected from each group). (C-D) Western blotting for expression of LC3B (C) and p62 (D) from the renal cortex in Control, DN, and DN plus different doses of catalpol-treated mice $(\mathrm{n}=3)$. Data represent the mean values \pm SD from three independent experiments, ${ }^{* *} p<0.01$ vs Control, $\# p<0.05$, \#\# $p$ $<0.01$ vs DN.

evidence that in an animal model of DN, treatment with catalpol ameliorated podocyte injury by stabilizing podocyte cytoskeleton structure and rescuing podocyte autophagy in DN.

Albuminuria, an earliest marker of $\mathrm{DN}$, which is related to podocyte injury in the progression of DN (Dinneen and Gerstein, 1997). Kidney hypertrophy, a common manifestation of DN patients, which is considered as an elevated kidney/body weight ratio (Bakris, 2008). In addition, glomerular mesangial expansion is another common pathological manifestation of
DN. In this study, we demonstrated that treatment with catalpol significant decreased albuminuria levels and improved kidney hypertrophy and mesangial expansion, providing evidence of catalpol efficacy, and these results were consistent with previous study (Dong and Chen, 2013). Although it has been reported that catalpol lowered blood glucose in diabetes mice (Li et al., 2014), in our study, a modest and non-significant decrease in blood glucose was observed in catalpol-treated mice, and thus, the renoprotection of catalpol was independent of blood glucose. 

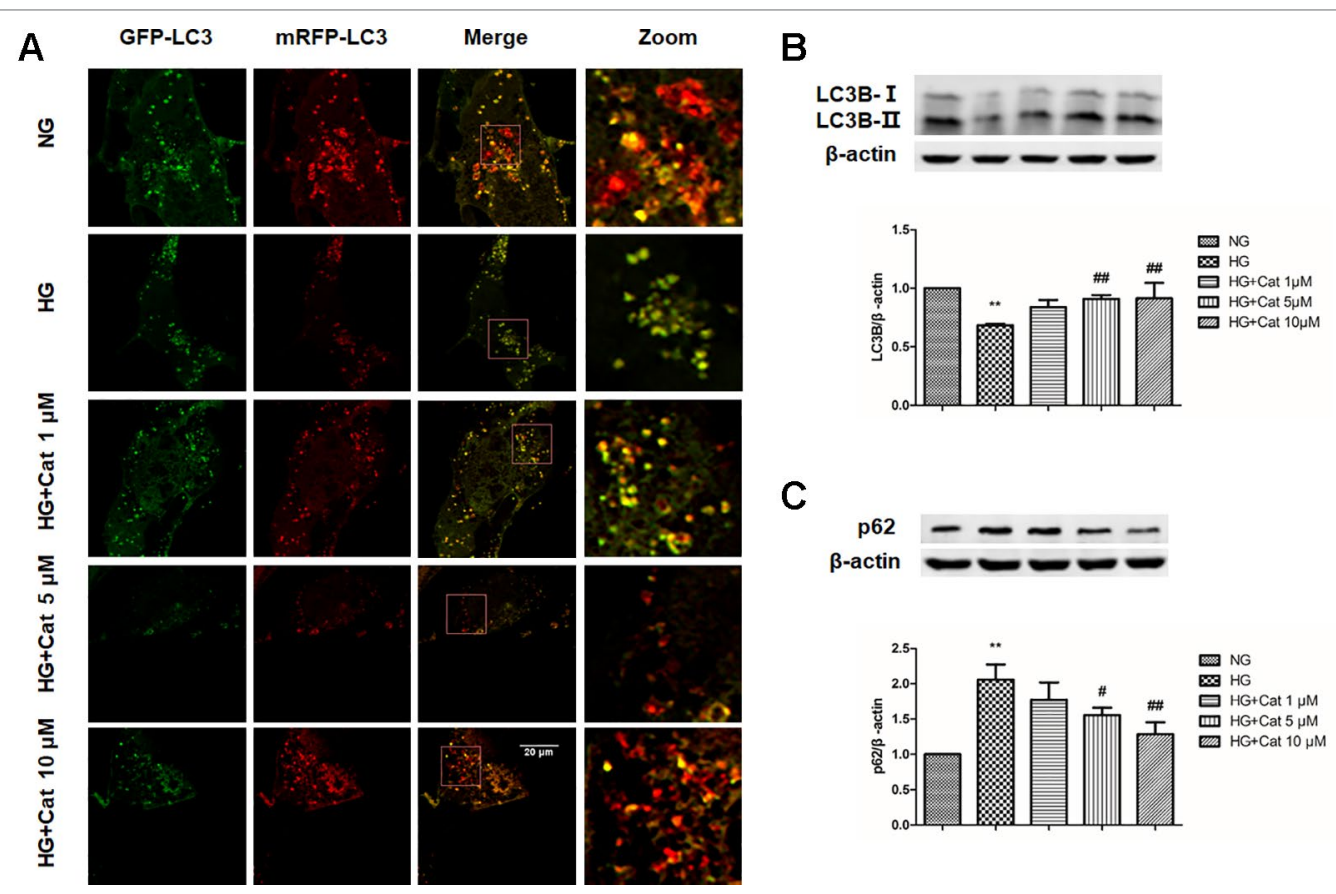

FIGURE 7 | Effect of catalpol on autophagy in podocytes exposed to high glucose. (A) Confocal laser scanning microscopy images of podocytes cultured with NG (5.5 mmol/L), HG (40 mmol/L), and HG plus different concentrations of catalpol (1, 5, $10 \mu \mathrm{M})$. Scale bar: $20 \mu \mathrm{m}$. (B-C) Western blotting for expression of LC3B (B) and p62 (C) from cultured podocytes in NG (5.5 mmol/L), HG (40 mmol/L), and HG plus different concentrations of catalpol (1, 5 , $10 \mu \mathrm{M})(\mathrm{n}=3)$. Data represent the mean values $\pm \mathrm{SD},{ }^{* *} p<0.01$ vs NG, ${ }^{\#} p<0.05$, ${ }^{\# \#} p<0.01$ vs HG.

In addition, there was a modest decline in creatinine clearance in DN mice, precluding evaluation of treatment effect on this factor.

Several studies indicated that drugs have protective effects on podocyte injury could improve the ability to ameliorate DN (Zhang et al., 2013; Kim et al., 2018). In this study, treatment with catalpol lowered the excretion of urinary podocyte marker podocalyxin, suggesting the protective effect of catalpol on podocyte injury in DN. Clearly, we observed a significant improvement in podocyte foot process effacement in catalpol-treated DN mice by using TEM. Nephrin, which is identified at the slit diaphragm of podocyte and is critical to maintain the integrity of filtration barrier (Trohatou et al., 2017). Synaptopodin, a actin-binding protein, which is located at podocyte cytoskeleton (Pan et al., 2018). When podocytes undergo injury in $\mathrm{DN}$, the expression of nephrin and synaptopodin were significantly decreased, our results showed that treatment with catalpol significantly increased the downregulation of nephrin and synaptopodin in $\mathrm{DN}$ mice. These results suggested that the renoprotection of catalpol was likely to be attributed to the improvement of podocyte injury in DN.

Podocyte injury accompanied by cytoskeleton rearrangement is observed in DN. Cytoskeleton rearrangement was closely associated with foot process effacement and albuminuria in several kidney diseases such as DN (Giganti and Friederich, 2003; Faul et al., 2008). Our results showed that catalpol could improve cytoskeleton disruption induced by HG, suggesting that catalpol improved podocyte injury by regulating the dynamics of the cytoskeleton. Recently study showed that, when podocytes undergo foot process effacement, podocytes shifted to a dynamic state from non-motile state in vivo (Brähler et al., 2016). In our study, the results strongly argue that catalpol suppressed podocyte migration induced by HG. These data demonstrated that catalpol protect podocyte injury through regulating the dynamics of the cytoskeleton and decreasing the motility of podocyte. Rho family of GTPases, particularly RhoA, Cdc42, and Racl are important molecular switches, which are closely related to dynamics of podocyte cytoskeleton (Wang et al., 2012). It has been shown that Podocyte-specific Racl deficiency promoted foot process effacement and proteinuria by disrupting cytoskeleton in DN mice (Scott et al., 2012; Blattner et al., 2013; Lv et al., 2018). In addition, the excessive activation of RhoA in podocytes resulted in foot process effacement and heavy proteinuria, accompanied by podocyte cytoskeleton disruption (Zhu et al., 2011; Wang et al., 2012). Therefore, controlled RhoA, Cdc42, and Racl activities are vital to stable podocyte cytoskeletons. In this study, our data revealed that catalpol could inhibit the excessive activation of RhoA and Cdc42 in podocytes induced by HG, suggesting that catalpol stabilized podocyte cytoskeleton through modulating the active forms of RhoA and Cdc42 but not Rac1.

Moreover, insufficient autophagy was observed specifically in podocytes of DN patients, which aggravated podocyte injury and proteinuria in DN. Thus, enhancing autophagy in podocytes is a potential treatment strategy for DN (Lenoir et al., 2015; Tagawa et al., 2016). It has been reported that catalpol promoted autophagy in liver fibrosis rats (Liu et al., 2018). In the present study, autophagosome numbers and 

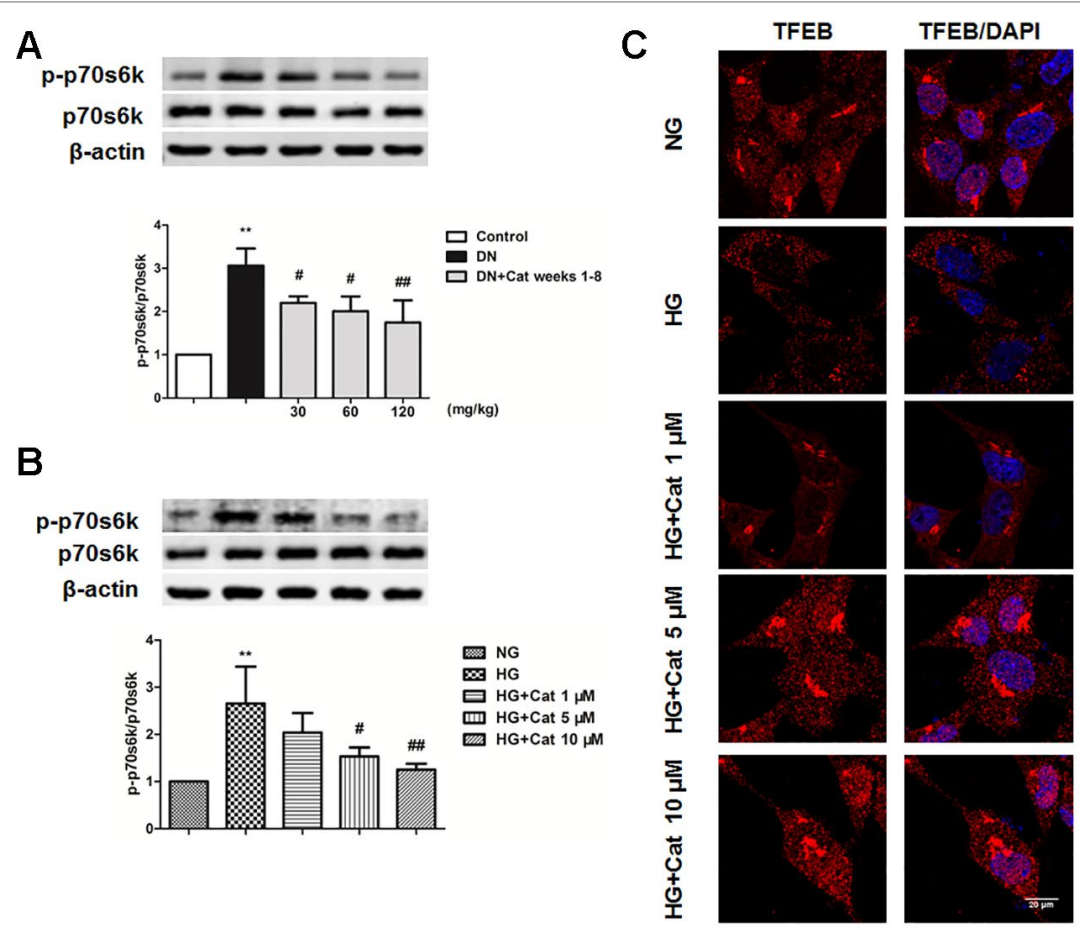

D

E
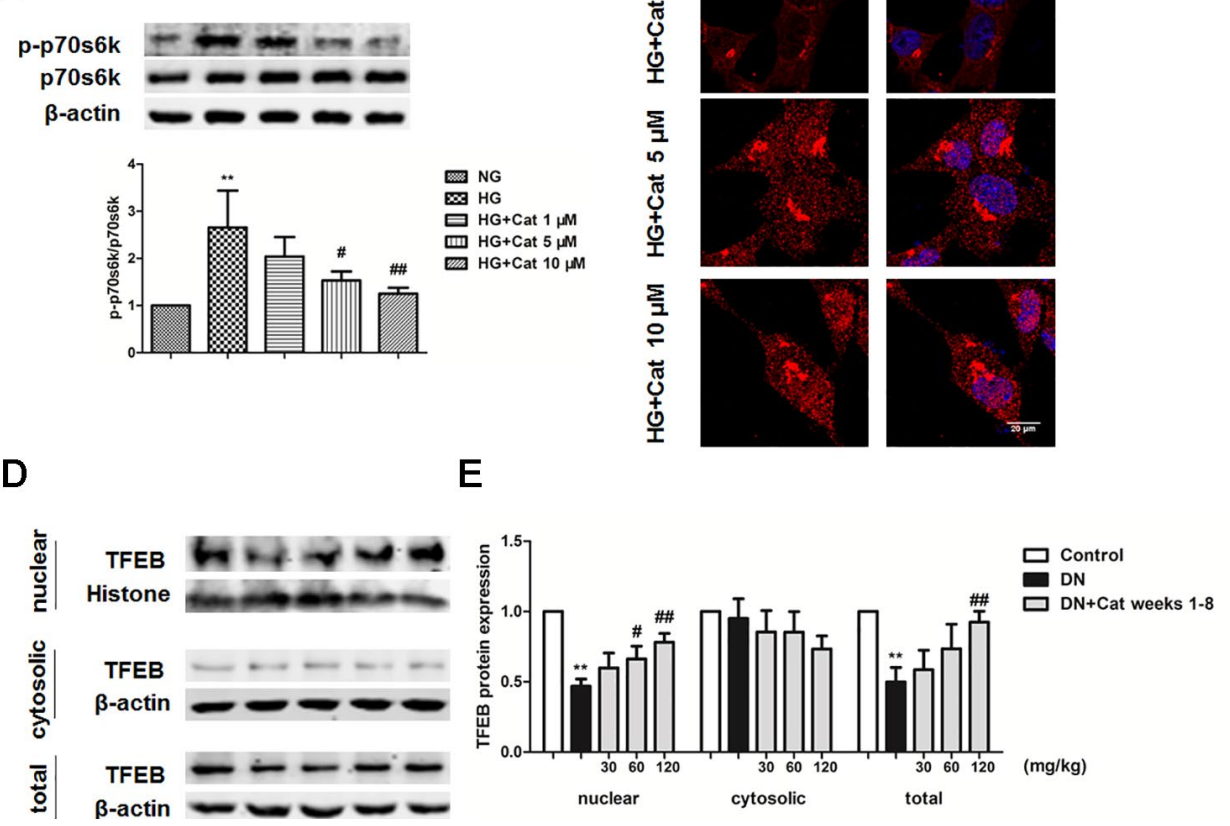

FIGURE 8 | Effects of catalpol on mTOR/TFEB pathway in DN mice and podocytes exposed to high glucose. (A) Representative blots show the expression of phosphorylated and total p70S6K in Control, DN, and DN plus catalpol-treated (30, 60, and 120 mg/kg, weeks 1-8) mice ( $\mathrm{n}=3$ ). (B) Representative blots show the expression of phosphorylated and total p70s6 k in podocytes cultured with NG (5.5 mmol/L), HG (40 mmol/L), and HG plus different concentrations of catalpol (1, 5, $10 \mu \mathrm{M})(\mathrm{n}=3)$. (C) Representative images of podocytes stained with TFEB antibody (red) and DAPI (blue). Scale bar: $20 \mu \mathrm{m}$. (D) Blots of the nuclear, cytosolic, and total TFEB expression in Control, DN, and DN plus different doses of catalpol-treated mice $(n=3)$. (E) Quantification of results in panel $D$. Data represent the mean values $\pm \mathrm{SD}$ from three independent experiments, ${ }^{* *} p<0.01$ vs Control, ${ }^{\#} p<0.05,{ }^{\# \#} p<0.01$ vs $\mathrm{DN}$.

LC3B expression were decreased, and p62 expression was increased in DN mice when compared with control mice, suggesting podocyte autophagy insufficiency in DN mice. In HG cultured podocytes, the number of autolysosome and the autophagy marker LC3B expression were decreased, and the p62 expression was increased, indicating that the autophagy flux was blocked in podocytes incubated with HG, while treatment with catalpol could improve podocyte autophagy in vivo and in vitro. These results demonstrated that catalpol improved podocyte autophagy insufficiency in DN. Furthermore, the exceeding activation of mTOR has been reported in podocytes in DN patients, which play a key role in the progression of DN (Gödel et al., 2011; Inoki et al., 2011). Moreover, TFEB was considered as the master regulator of autophagy (Settembre et al., 2011). It has been reported that the nuclear translocation of TFEB is inhibited by mTOR activation, and inhibition of mTOR activity could promote TFEB nuclear translocation to protect podocyte injury in DN (Martina et al., 2012; Zhao et al., 2018). Our results suggested that mTOR was hyperactive, and accompanied with the decreased nuclear translocation of TFEB in vivo and in vitro, treatment with catalpol inhibited mTOR activity and promoted TFEB nuclear translocation in DN. These results suggested that catalpol enhanced podocyte autophagy through inhibiting mTOR activity to promote TFEB nuclear translocation, and subsequently enhanced autophagy.

\section{CONCLUSION}

In conclusion, our study demonstrated that catalpol could improve podocyte injury in $\mathrm{DN}$, and the protective effect of catalpol might be attributed to the stabilization of podocyte 
cytoskeleton structure and the improvement of impaired podocyte autophagy.

\section{DATA AVAILABILITY STATEMENT}

All datasets generated for this study are included in the article/ supplementary material.

\section{ETHICS STATEMENT}

All animal experiments were approved by the Institutional Animal Care and Research Ethics Committee of Henan University of Chinese Medicine and conformed to the guidelines of the National Institute of Health for the Care and Use of Laboratory Animals.

\section{AUTHOR CONTRIBUTIONS}

$\mathrm{YC}, \mathrm{QL}$, and $\mathrm{XZ}$ contributed to the experiment design and manuscript writing. YC, QL, ZS, WM, YZ, ML, and BW

\section{REFERENCES}

Bakris, G. L. (2008). Slowing nephropathy progression: focus on proteinuria reduction. Clin. J. Am. Soc Nephrol. 31, S3-S10. doi: 10.2215/CJN03250807

Blattner, S. M., Hodgin, J. B., Nishio, M., Wylie, S. A., Saha, J., Soofi, A. A., et al. (2013). Divergent functions of the Rho GTPases Racl and Cdc42 in podocyte injury. Kidney Int. 84, 920-930. doi: 10.1038/ki.2013.175

Brähler, S., Yu, H., Suleiman, H., Krishnan, G. M., Saunders, B. T., Kopp, J. B., et al. (2016). Intravital and kidney slice imaging of podocyte membrane dynamics. J. Am. Soc Nephrol. 27, 3285-3290. doi: 10.1681/ASN.2015121303

Chen, W., Li, X., Jia, L. Q., Wang, J., Zhang, L., Hou, D., et al. (2013). Neuroprotective activities of catalpol against CaMKII-dependent apoptosis induced by LPS in PC12 cells. Br. J. Pharmacol. 169, 1140-1152. doi: 10.1111/bph.12200

Cho, N. H., Shaw, J. E., Karuranga, S., Huang, Y., da Rocha Fernandes, J. D., Ohlrogge, A. W., et al. (2018). IDF Diabetes Atlas: Global estimates of diabetes prevalence for 2017 and projections for 2045. Diabetes Res. Clin. Pract. 138, 271-281. doi: 10.1016/j.diabres.2018.02.023

Dai, H., Liu, Q., and Liu, B. (2017). Research progress on mechanism of podocyte depletion in diabetic nephropathy. J. Diabetes. Res. 2017, 2615286. doi: $10.1155 / 2017 / 2615286$

Denhez, B., Rousseau, M., Dancosst, D. A., Lizotte, F., Guay, A., Auger-Messier, M., et al. (2019). Diabetes-induced DUSP4 reduction promotes podocyte dysfunction and progression of diabetic nephropathy. Diabetes 68, 1026-1039. doi: $10.2337 / \mathrm{db} 18-0837$

Dinneen, S. F., and Gerstein, H. C. (1997). The association of microalbuminuria and mortality in non-insulin-dependent diabetes mellitus. A systematic overview of the literature. Arch. Intern. Med. 157, 1413-1418. doi: 10.1001/ archinte.1997.00440340025002

Dong, Z., and Chen, C. X. (2013). Effect of catalpol on diabetic nephropathy in rats. Phytomedicine 20, 1023-1029. doi: 10.1016/j.phymed.2013.04.007

Faul, C., Donnelly, M., Merscher-Gomez, S., Chang, Y. H., Franz, S., Delfgaauw, J., et al. (2008). The actin cytoskeleton of kidney podocytes is a direct target of the antiproteinuric effect of cyclosporine A. Nat. Med. 14, 931-938. doi: 10.1038/ nm. 1857

Fineberg, D., Jandeleit-Dahm, K. A., and Cooper, M. E. (2013). Diabetic nephropathy: diagnosis and treatment. Nat. Rev. Endocrinol. 9, 713-723. doi: 10.1038/nrendo.2013.184 performed the experiments. YC and QL analyzed the data. XZ and WF supervised the project. All authors read and approved the final manuscript.

\section{FUNDING}

This study was supported by The National Key Research and Development Project (The Major Project for Research of the Modernization of TCM): Key Technology Research for the Characteristic Chinese Medicine Industry Chain of Rehmannia glutinosa (2017YFC1702800) and The Major Science and Technology Projects in Henan Province: Study on the key technology for quality control and the key characteristics of $R$. glutinosa, Dioscorea opposita Thunb, and Achyranthes bidentata Blume from Henan Province (171100310500).

\section{ACKNOWLEDGMENTS}

We thank the Co-construction Collaborative Innovation Center for Chinese Medicine and Respiratory Diseases by Henan \& Education Ministry of P.R. China, Zhengzhou, China (2013638).

Garcia-Mata, R., Wennerberg, K., Arthur, W. T., Noren, N. K., Ellerbroek, S. M., and Burridge, K. (2006). Analysis of activated GAPs and GEFs in cell lysates. Methods Enzymol. 406, 425-437. doi: 10.1016/S0076-6879(06) 06031-9

George, B., Verma, R., Soofi, A. A., Garg, P., Zhang, J., Park, T. J., et al. (2012). Crk1/2-dependent signaling is necessary for podocyte foot process spreading in mouse models of glomerular disease. J. Clin. Invest. 122, 674-692. doi: 10.1172/JCI60070

Georgianos, P. I., and Agarwal, R. (2017). Endothelin A receptor antagonists in diabetic kidney disease. Curr. Opin. Nephrol. Hypertens. 26, 338-344. doi: 10.1097/MNH.0000000000000342

Giganti, A., and Friederich, E. (2003). The actin cytoskeleton as a therapeutic target: state of the art and future directions. Prog. Cell Cycle Res. 5, 511-525.

Ginet, V., Spiehlmann, A., Rummel, C., Rudinskiy, N., Grishchuk, Y., LuthiCarter, R., et al. (2014). Involvement of autophagy in hypoxic-excitotoxic neuronal death. Autophagy 10, 846-860. doi: 10.4161/auto.28264

Gödel, M., Hartleben, B., Herbach, N., Liu, S., Zschiedrich, S., Lu, S., et al. (2011). Role of mTOR in podocyte function and diabetic nephropathy in humans and mice. J. Clin. Invest. 121, 2197-2209. doi: 10.1172/JCI44774

Hara, M., Oohara, K., Dai, D. F., and Liapis, H. (2019). Mitotic catastrophe causes podocyte loss in the urine of human diabetics. Am. J. Pathol. 189, 248-257. doi: 10.1016/j.ajpath.2018.10.016

Hartleben, B., Gödel, M., Meyer-Schwesinger, C., Liu, S., Ulrich, T., Köbler, S., et al. (2010). Autophagy influences glomerular disease susceptibility and maintains podocyte homeostasis in aging mice. J. Clin. Invest. 120, 1084-1096. doi: 10.1172/JCI39492

Huang, Z., Peng, Y., Yu, H., Yu, X., Zhou, J., and Xiao, J. (2018). RhoA protects the podocytes against high glucose-induced apoptosis through YAP and plays critical role in diabetic nephropathy. Biochem. Biophys. Res. Commun. 504, 949-956. doi: 10.1016/j.bbrc.2018.08.204

Inoki, K., Mori, H., Wang, J., Suzuki, T., Hong, S., Yoshida, S., et al. (2011). mTORC1 activation in podocytes is a critical step in the development of diabetic nephropathy in mice. J. Clin. Invest. 121, 2181-2196. doi: 10.1172/ JCI44771

Jefferson, J. A., Alpers, C. E., and Shankland, S. J. (2011). Podocyte biology for the bedside. Am. J. Kidney Dis. 58, 835-845. doi: 10.1053/j.ajkd.2011. 03.033 
Jin, J., Tu, Q., Gong, J., Zhao, L., Liang, S., and He, Q. (2019). Autophagy activity and expression pattern of autophagy-related markers in the podocytes of patients with lupus nephritis: association with pathological classification. Ren. Fail. 41, 294-302. doi: 10.1080/0886022X.2019.1598432

Kikuchi, Y., Yamada, M., Imakiire, T., Kushiyama, T., Higashi, K., Hyodo, N., et al. (2007). A Rho-kinase inhibitor, fasudil, prevents development of diabetes and nephropathy in insulin-resistant diabetic rats. J. Endocrinol. 192, 595-603. doi: 10.1677/JOE-06-0045

Kim, D. Y., Kang, M. K., Lee, E. J., Kim, Y. H., Oh, H., and Kang, Y. H. (2018). Eucalyptol inhibits advanced glycation end products-induced disruption of podocyte slit junctions by suppressing RAGE-ERK-c-Myc signaling pathway. Mol. Nutr. Food Res. 62, e1800302. doi: 10.1002/mnfr.201800302

Kume, S., and Koya, D. (2015). Autophagy: a novel therapeutic target for diabetic nephropathy. Diabetes Metab. J. 39, 451-460. doi: 10.4093/dmj.2015.39.6.451

Lal, M. A., and Patrakka, J. (2018). Understanding podocyte biology to develop novel kidney therapeutics. Front. Endocrinol. 9, 409. doi: 10.3389/fendo.2018.00409

Lenoir, O., Jasiek, M., Hénique, C., Guyonnet, L., Hartleben, B., Bork, T., et al. (2015). Endothelial cell and podocyte autophagy synergistically protect from diabetes-induced glomerulosclerosis. Autophagy 11, 1130-1145. doi: 10.1080/15548627.2015.1049799

Li, J. J., Kwak, S. J., Jung, D. S., Kim, J. J., Yoo, T. H., Ryu, D. R., et al. (2007). Podocyte biology in diabetic nephropathy. Kidney Int. Suppl. 72, S36-S42. doi: 10.1038/sj.ki.5002384

Li, X., Xu, Z., Jiang, Z., Sun, L., Ji, J., Miao, J., et al. (2014). Hypoglycemic effect of catalpol on high-fat diet/streptozotocin-induced diabetic mice by increasing skeletal muscle mitochondrial biogenesis. Acta Biochim. Biophys. Sin. 46, 738748. doi: 10.1093/abbs/gmu065

Lin, J. S., and Susztak, K. (2016). Podocytes: the Weakest Link in Diabetic Kidney Disease? Curr. Diab. Rep. 16, 45. doi: 10.1007/s11892-016-0735-5

Lin, T. A., Wu, V. C., and Wang, C. Y. (2019). Autophagy in Chronic Kidney Diseases. Cells 8, 61. doi: 10.3390/cells8010061

Liu, Z., Zhu, P., Zhang, L., Xiong, B., Tao, J., Guan, W., et al. (2018). Autophagy inhibition attenuates the induction of anti-inflammatory effect of catalpol in liver fibrosis. Biomed. Pharmacother. 103, 1262-1271. doi: 10.1016/j.biopha.2018.04.156

Looker, H. C., Mauer, M., Saulnier, P. J., Harder, J. L., Nair, V., Boustany-Kari, C. M., et al. (2019). Najafian, b. Changes in albuminuria but not gfr are associated with early changes in kidney structure in type 2 diabetes. J. Am. Soc Nephrol. 30 , 1049-1059. doi: 10.1681/ASN.2018111166

Lv, Z., Hu, M., Fan, M., Li, X., Lin, J., Zhen, J., et al. (2018). Podocyte-specific Racl deficiency ameliorates podocyte damage and proteinuria in STZ-induced diabetic nephropathy in mice. Cell Death Dis. 9, 342. doi: 10.1038/s41419-018-0353-Z

Martina, J. A., Chen, Y., Gucek, M., and Puertollano, R. (2012). MTORC1 functions as a transcriptional regulator of autophagy by preventing nuclear transport of TFEB. Autophagy 8, 903-914. doi: 10.4161/auto.19653

Mizushima, N., and Komatsu, M. (2011). Autophagy: renovation of cells and tissues. Cell 147, 728-741. doi: 10.1016/j.cell.2011.10.026

Mizushima, N., Levine, B., Cuervo, A. M., and Klionsky, D. J. (2008). Autophagy fights disease through cellular self-digestion. Nature 451, 1069-1075. doi: 10.1038 /nature 06639

Molitch, M. E., Steffes, M., Sun, W., Rutledge, B., Cleary, P., de Boer, I. H., et al. (2010). Epidemiology of diabetes interventions and complications study group. Development and progression of renal insufficiency with and without albuminuria in adults with type 1 diabetes in the diabetes control and complications trial and the epidemiology of diabetes interventions and complications study. Diabetes Care 33, 1536-1543. doi: 10.2337/dc09-1098

Mundel, P., Reiser, J., Zúñiga Mejía Borja, A., Pavenstädt, H., Davidson, G. R., Kriz, W., et al. (1997). Rearrangements of the cytoskeleton and cell contacts induce process formation during differentiation of conditionally immortalized mouse podocyte cell lines. Exp. Cell Res. 236, 248-258. doi: 10.1006/excr.19973739

Nagata, M. (2016). Podocyte injury and its consequences. Kidney Int. 89, 1221-1230. doi: 10.1016/j.kint.2016.01.012

Pan, Y., Jiang, S., Hou, Q., Qiu, D., Shi, J., Wang, L., et al. (2018). Dissection of glomerular transcriptional profile in patients with diabetic nephropathy: SRGAP2a protects podocyte structure and function. Diabetes 67, 717-730. doi: $10.2337 / \mathrm{db} 17-0755$

Ravindran, S., Pasha, M., Agouni, A., and Munusamy, S. (2019). Microparticles as potential mediators of high glucose-induced renal cell injury. Biomolecules 9 , 348. doi: 10.3390/biom 9080348
Reiser, J., and Sever, S. (2013). Podocyte biology and pathogenesis of kidney disease. Annu. Rev. Med. 64, 357-366. doi: 10.1146/annurev-med-050311-163340

Scott, R. P., Hawley, S. P., Ruston, J., Du, J., Brakebusch, C., Jones, N., et al. (2012). Podocyte-specific loss of Cdc42 leads to congenital nephropathy. J. Am. Soc Nephrol. 23, 1149-1154. doi: 10.1681/ASN.2011121206

Settembre, C., Di Malta, C., Polito, V. A., Arencibia, Garcia, Vetrini, F., Erdin, S., et al. (2011). TFEB links autophagy to lysosomal biogenesis. Science 332, 14291433. doi: $10.1126 /$ science. 1204592

Settembre, C., Zoncu, R., Medina, D. L., Vetrini, F., Erdin, S., Erdin, S., et al. (2012). A lysosome-to-nucleus signalling mechanism senses and regulates the lysosome via mTOR and TFEB. EMBO J. 31, 1095-1108. doi: 10.1038/ emboj. 2012.32

Settembre, C., De Cegli, R., Mansueto, G., Saha, P. K., Vetrini, F., Visvikis, O., et al. (2013). TFEB controls cellular lipid metabolism through a starvation-induced autoregulatory loop. Nat. Cell Biol. 15, 647-658. doi: 10.1038/ncb2718

Shankland, S. J. (2006). The podocyte's response to injury: role in proteinuria and glomerulosclerosis. Kidney Int. 69, 2131-2147. doi: 10.1038/sj.ki.5000410

Tagawa, A., Yasuda, M., Kume, S., Yamahara, K., Nakazawa, J., Chin-Kanasaki, M., et al. (2016). Impaired podocyte autophagy exacerbates proteinuria in diabetic nephropathy. Diabetes 65, 755-767. doi: 10.2337/db15-0473

Toffoli, B., Zennaro, C., Winkler, C., Giordano Attianese, G. M. P., Bernardi, S., Carraro, M., et al. (2018). Hemicentin 1 influences podocyte dynamic changes in glomerular diseases. Am. J. Physiol. Renal. Physiol. 314, F1154-F1165. doi: 10.1152/ajprenal.001982017

Trohatou, O., Tsilibary, E. F., Charonis, A., Iatrou, C., and Drossopoulou, G. (2017). Vitamin D3 ameliorates podocyte injury through the nephrin signalling pathway. J. Cell. Mol. Med. 21, 2599-2609. doi: 10.1111/jcmm.13180

Tryggvason, K., Patrakka, J., and Wartiovaara, J. (2006). Hereditary proteinuria syndromes and mechanisms of proteinuria. N. Engl. J. Med. 354, 1387-1401. doi: 10.1056/NEJMra052131

Wang, L., Ellis, M. J., Gomez, J. A., Eisner, W., Fennell, W., Howell, D. N., et al. (2012). Mechanisms of the proteinuria induced by Rho GTPases. Kidney Int. 81, 1075-1085. doi: 10.1038/ki.2011.472

Wiggins, R. C. (2007). The spectrum of podocytopathies: a unifying view of glomerular diseases. Kidney Int. 71, 1205-1214. doi: 10.1038/sj.ki.5002222

Yan, J., Wang, C., Jin, Y., Meng, Q., Liu, Q., Liu, Z., et al. (2018). Catalpol ameliorates hepatic insulin resistance in type 2 diabetes through acting on AMPK/NOX4/PI3K/AKT pathway. Pharmacol. Res. 130, 466-480. doi: 10.1016/j.phrs.2017.12.026

Yasuda-Yamahara, M., Kume, S., Tagawa, A., Maegawa, H., and Uzu, T. (2015). Emerging role of podocyte autophagy in the progression of diabetic nephropathy. Autophagy 11, 2385-2386. doi: 10.1080/15548627.2015.1115173

Ye, B., Wang, Q., Hu, H., Shen, Y., Fan, C., Chen, P., et al. (2019). Restoring autophagic flux attenuates cochlear spiral ganglion neuron degeneration by promoting TFEB nuclear translocation via inhibiting MTOR. Autophagy 15, 998-1016. doi: 10.1080/15548627.2019.1569926

Zhang, L., Li, R., Shi, W., Liang, X., Liu, S., Ye, Z., et al. (2013). NFAT2 inhibitor ameliorates diabetic nephropathy and podocyte injury in $\mathrm{db} / \mathrm{db}$ mice. $\mathrm{Br}$. J. Pharmacol. 170, 426-439. doi: 10.1111/bph.12292

Zhao, X., Chen, Y., Tan, X., Zhang, L., Zhang, H., Li, Z., et al. (2018). Advanced glycation end-products suppress autophagic flux in podocytes by activating mammalian target of rapamycin and inhibiting nuclear translocation of transcription factor EB. J. Pathol. 245, 235-248. doi: 10.1002/path5077

Zhu, L., Jiang, R., Aoudjit, L., Jones, N., and Takano, T. (2011). Activation of RhoA in podocytes induces focal segmental glomerulosclerosis. J. Am. Soc Nephrol. 22, 1621-1630. doi: 10.1681/ASN.2010111146

Conflict of Interest: The authors declare that the research was conducted in the absence of any commercial or financial relationships that could be construed as a potential conflict of interest.

Copyright $\odot 2019$ Chen, Liu, Shan, Mi, Zhao, Li, Wang, Zheng and Feng. This is an open-access article distributed under the terms of the Creative Commons Attribution License (CC BY). The use, distribution or reproduction in other forums is permitted, provided the original author(s) and the copyright owner(s) are credited and that the original publication in this journal is cited, in accordance with accepted academic practice. No use, distribution or reproduction is permitted which does not comply with these terms. 